\title{
Méprisé, mais pas méprisable - échec de la rhétorique de dissuasion dans Demain matin, Montréal m'attend de Michel Tremblay
}

\author{
Abstract \\ Despised but not Despicable - Failure of the Rhetoric of Dissuasion \\ in Demain matin, Montréal m'attend by Michel Tremblay
}

In Demain matin, Montréal m'attend, a comedy by Michel Tremblay written in the era of a Quiet Revolution, contempt takes on an argumentative value. Lola Lee, a singer and artist in La Main - an artistic neighborhood in Montreal. Lola's younger sister - Louise - is jealous of the popularity gained by her older sibling. Lola Lee, in the attempt to discourage Louise from the idea of following in her footsteps travels with her through the most infamous artistic venues. During this trip Louise meets artists with questionable morals. The aim of the article is firstly to show how this journey, instead of generating contempt, becomes the core of searching for your own artistic identity. Secondly, the article explains how Tremblay's art, using metaphors in the style of Almodóvar, represent the national dramaturgy of Quebec, in which the hero, which is an allegory of the whole society, undergoes an evolution that allows him to appreciate his own identity instead of despising it.

Keywords: Michel Tremblay, Canadian theater, theater of Quebec, rhetoric

Mots-clés: Michel Tremblay, théâtre canadien, théâtre québécois, rhétorique

Dans les années 1970, la dramaturgie canadienne d'expression française, et plus particulièrement québécoise, «malgré la multiplication des auteurs et l'éclatement de formes dramatiques $»^{1}$, est dominée par l'œuvre de Michel Tremblay. Comme l'explique le dramaturge dans une interview, le thème récurrent de ses ouvrages, c'est la nostalgie et la réussite sociale, presque impossible à réaliser

\footnotetext{
1 M. Greffard, J.-G. Sabourin, Le Théâtre québecois, Québec 1997, p. 78.
} 
à cause de «toutes les misères du monde » qui s'y opposent ${ }^{2}$. Dans Demain matin, Montréal m 'attend, une comédie écrite dans les années 1970, revient aussi le motif des rêves que les personnages cherchent à réaliser. En effet, Lola Lee, une chanteuse et artiste de La Main, un quartier montréalais à statut mythique, jalouse de la popularité éventuelle de sa sœur cadette, essaie de la décourager de suivre ses traces en la faisant traverser les endroits les plus dédaigneux et en la faisant connaître les artistes très peu glorieux. La présente étude se propose, dans un premier temps, d'analyser comment la rhétorique par anti-modèle aboutit à une fin inattendue et, dans un deuxième temps, d'expliquer comment cette pièce de Tremblay est représentative sur le plan des changements sociaux et identitaires qui se sont produits peu avant sa création, lors de la Révolution tranquille.

Depuis qu'il a été écrit, le texte de Demain matin, Montréal m'attend a été mis en scène plusieurs fois (en 1972, il entre dans le répertoire du Théâtre Maisonneuve, du Grand Théâtre de Québec et du Centre National des Arts, en 1995, Denise Filiatrault offre une nouvelle mise en scène) et jusqu'à nos jours, il jouit d'une popularité inébranlable du public. En 2017, la comédie est montée sur scène dans le cadre de la $29^{\circ}$ édition du festival Francofolies à Montréal, et en même année, devient la production du Théâtre du Nouveau Monde. Bien que le texte date de 1970, il suscite toujours un intérêt tout particulier parce que, comme l'affirme l'actrice Marie-Andrée Lemieux, « les sujets et les propos de cette œuvre-là ne vieilliront jamais $»^{3}$. On ne peut contredire cet avis parce que, même si l'action se déroule dans un passé qui nous semble un peu vieillot, le texte de Michel Tremblay évoque une quête toujours actuelle : celle de la célébrité.

La problématique de l'ouvrage se situe aux abords du monde du music-hall, on y en retrouve la description drôle et tragique : ses vedettes, ses victimes, ses réussites, ses déboires. Le texte foisonne de personnages, mais l'histoire tourne avant tout autour de Louise Tétrault, jeune femme de Saint-Martin en banlieue de Montréal, et de sa sœur Rita, alias Lola Lee. Louise Tétrault, en effet, une jeune chanteuse de campagne de dix-huit ans, qui rêve de partir à Montréal, s'inscrit au concours amateur. Elle gagne le trophée pour sa performance musicale et dit adieu à sa mère, à son travail et à sa vie. Elle a envie de chanter. Elle va à Montréal pour rejoindre sa sœur qui a déjà gagné ce concours il y a quinze ans et qui est à présent une star du milieu underground montréalais, connue sous le pseudonyme de Lola Lee. Elle a mis de longues années pour gagner la position artistique que Louise, à son avis, cherche à occuper sur-le-champ. Lola est jalouse de sa carrière qu'elle doit à son talent : " Chus venue au music-hall parce que j'avais du talent, pas juste pour montrer mes desses pis mon pataclan au monde $»^{4}$. Elle voit donc Louise comme une menace, et, pour la décourager et prouver que la voie de la réussite est épineuse et compliquée, décide de lui faire voir le deuxième côté de la médaille. Elle l'amène dans la Main, c'est-à-dire le boulevard Saint-Laurent, centre culturel montréalais, pour lui montrer à quel point c'est une mauvaise idée de venir

2 L. Boulanger, Pièces à conviction. Entretiens avec Michel Tremblay, Ottawa 2001, p. 45.

3 Lors d'un entretien radiophonique à la Radio VM, le 27 septembre 2017, in https://www. youtube.com/watch?v=K9GjQiVYxbQ date de la consultation.

4 M. Tremblay, Demain matin, Montréal m'attend, « Théâtre II », Montréal 2006, p. 106. 
à Montréal pour y chercher une célébrité. Elles vont donc dans un bar gay, où Louise est un peu ébranlée en voyant des hommes s'habillant en femme, elle fait connaissance avec des prostituées, mais elle ne se laisse pas déstabiliser. Elle veut être chanteuse en imitant Lola, elle est persuadée que sa sœur va l'aider à interpréter ses chansons d'une façon majestueuse. Malheureusement pour Louise, sa sœur Lola ne veut pas entendre que Louise devienne une star (« laisse-moi te dire que ta carrière d'imitatrice vient de s'éteindre $»^{5}$ ) pour deux raisons : Louise représente pour elle « un mauvais souvenir » et elle voit en sa sœur « une jeune et dangereuse compétitrice dans ce monde sans pitié $\iota^{6}$. Elle entend, en effet, prendre toutes les précautions pour l'en dissuader et l'incite, par conséquent, à descendre de plus en plus bas dans ce milieu méprisé par la bonne société.

Dans son écriture dramatique, Tremblay joue sur plusieurs formes (à savoir il « opère une résurgence du chœur, s'inspire de la revue, se réfère à d'autres œuvres $\gg^{7}$ ). Dans le cas de Demain matin, un rôle particulier est attribué aux chansons qui ont été ajoutées à travers les versions successives de la pièce. Les textes des chansons, en effet, font partie de l'intrigue et y apportent des éléments complémentaires. Lola, par exemple, s'en sert pour présenter à Louise le milieu artistique montréalais d'une manière que celle-ci le méprise. Afin de dissuader sa sœur cadette d'y accéder, Lola Lee met alors en évidence la nature apparente et illusoire de tout lien amical et amoureux des membres de cette société :

Du premier au dernier.

Du plus blond au plus noir.

Plus qu'y sont beaux.

Plus qu'y faut que t'en méfies !

Quand ces gars-là veulent s'approcher.

C'est jamais pour t'aimer!

$\mathrm{Y}$ te font souffrir, Louise, oui, y te font souffrir.

Pis là y s'mettent à te trouver plate !

Regarde autour de toi

(...)

T'es ben tu-seule.

Quand tu te couches le soir dans ton lit $!^{8}$

Lola Lee cherche, en effet, à dévoiler à sa sœur le revers de la médaille du showbiz. Elle espère ainsi provoquer en elle du dégoût, de l'aversion et du mépris pour couper court à ses projets. Commence alors la tournée de La Main. Après le Bolivar Lounge (le club érotique où elle brille), Lola entraînera sa petite sœur au Meat Rack (un bar spécialisé pour travestis), puis au bordel-cabaret de Betty Bird. Ainsi, le lecteur assiste à une véritable virée en ville, fait la connaissance des personnages dont les joies et peines sont mises en lumière. Tous ces êtres en crise d'identité vont, d'après le projet de Lola, révéler à Louise des zones d'ombre de la condition humaine. Par l'intermédiaire des anti-exemples, elle met en œuvre une

\footnotetext{
5 Ibid., p. 111.

6 G. Desmeules, Ch. Lahaie, Les Personnages du théâtre québecois, Québec 2000, p. 28.

7 M. Greffard, J.-G. Sabourin, op. cit., p. 80.

8 M. Tremblay, op. cit., p. 121.
} 
rhétorique dissuasive, et par la présentation des réalités perverses, elle cherche à persuader Louise que l'univers auquel elle aspire est digne du mépris. Lola Lee que Louise admire pour avoir réussi dans le monde du showbiz, devient un modèle à suivre et, dans la dimension rhétorique, elle devient, pour se servir de la définition de Chaïm Perelman, "l'argument d'autorité », (argumentum ad verecundiam), « elle sert de caution à l'action envisagée ${ }^{9}$. Il s'agit, en effet, d'un modèle qu'on admire, qui a de l'autorité ou qui se distingue par son prestige social, sa compétence ou sa fonction. Par conséquent, l'argument par autorité prétend à la démonstration de ce modèle. Pour Louise, Lola est une personnalité célèbre, réputée et sage, digne d'être imitée. Dans l'argumentation par modèle, en effet, « si l'inférieur qui imite le modèle a quelque tendance à lui ressembler, il la rend, par le fait même, moins distinguée $»^{10}$. Par conséquent, permettre à Louise d'imiter son talent, son personnage scénique, signifierait pour Lola une dépréciation professionnelle. Comme l'explique Perelman, «si l'inférieur imite le supérieur, ce dernier ne désire, pour rien au monde, ressembler à l'inférieur ${ }^{11}$. Ayant créé, sans le vouloir, aux yeux de Louise, l'èthos de la sœur - artiste qui a tout réussi sur le plan professionnel, il ne laisse pas d'autre choix à Lola que de jouer sur le pathos, donc sur « l'ensemble des émotions, passions et sentiments que l'orateur doit susciter dans son auditoire grâce à son discours $\rangle^{12}$. L'auditeur, dans ce cas, c'est Lola, son auditoire, Louise et le discours est remplacé par les images négatives reçues par Louise lors du trajet effectué dans des clubs montréalais.

Louise observe, en effet, des jalousies et de petites haines, comme par exemple celles qui surgissent dans la conversation, au Meat Rack, entre deux protagonistes qui font partie de l'univers artistique de La Main, Duchesse de Langeais (qui est aussi le héros éponyme d'une autre pièce de Tremblay, publiée en 1968) et Marcel Gérard :

LA DUCHESSE - Je suis très heureuse d'être de retour parmi vous.

MARCEL GÉRARD - Ouan, ben pas nous autres ! (...)

LA DUCHESSE - J'ai un panier d'ananas, de bananes et de beaux rutabagas...

MARCEL GÉRARD - Ça vaut la peine ! Ça vaut la peine d'aller passer un an au Mexique pour rev'nir juste plus vieille! (...)

LA DUCHESSE - Y parait que pendant que j'vieillissais au Mexique, tu te rendais ridicule à Montréal, toi ? (...) Y paraît que tu rêvais de scandales internationaux pis que tu te retrouvais toujours dans des p'tits potins locaux, la mine basse pis le nez dans'poussière $!^{13}$

Ce déplacement spatial de l'action et des personnages principaux offre, certes, l'avantage de relancer la pièce par la multiplication des décors, des anecdotes et des atmosphères. Mais il a aussi pour effet de conférer aux lieux une valeur symbolique qui en fait des personnages à part entière, dans la mesure où ils participent à l'éclatement du drame ou à son dénouement et, avant tout, permettent à la protagoniste de rechercher sa propre identité. Dès le début du premier acte,

\footnotetext{
9 Ch. Perelman, L'Empire rhétorique, Paris 2012, p. 140.

${ }_{10}$ Ibid., p. 141.

11 Ibid.

12 O. Reboul, Introduction à la rhétorique, Paris 1995, p. 60.

13 M. Tremblay, op. cit., p. 116-117.
} 
les deux protagonistes, Louise et Lola, se métamorphosent en faire-valoir de La Main, ce qui réduit momentanément leur rôle au rang de prétexte. Cela provoque un déséquilibre que les critiques ont souvent assimilé à un manque d'intensité dramatique, mais en même temps ce processus joue un rôle important dans la confrontation des personnages avec le monde externe (plus ou moins méprisé), une rencontre, qui, elle, les fait revêtir une identité personnelle.

Mais en réalité, la situation est plus complexe, car le déplacement, dans Demain matin, Montréal m'attend est soumis à une double logique et revêt deux conséquences pour les protagonistes principales. Dans le cas de Louise, les trois lieux visités marquent les phases de ce qu'on peut bien qualifier de voyage initiatique, de descente dans les entrailles de l'enfer moral. Il s'agit là d'une quête imposée par Lola à Louise, dans le but de la mettre littéralement à l'épreuve en la confrontant à une réalité de moins en moins supportable et en éveillant un mépris de plus en plus intense.

Pourtant, on remarque que, ce qui, chez Louise, n'est qu'un voyage initiatique - aussi pénible soit-il - a des effets beaucoup plus dévastateurs chez Lola, puisqu'il correspond à une régression symbolique dans son cas. Lola refait symboliquement, mais à rebours, le chemin qu'elle a péniblement parcouru en douze ans, le chemin qui l'a fait arriver au point où elle se trouve, le chemin que Louise doit à tout prix mépriser. Or, l'univers de Lola est rempli des jalousies, trahisons et frustrations. Pas de relation, pas d'amitié. Lorsque les projecteurs s'éteignent et que le spectacle est terminé, les protagonistes se lancent des répliques assassines pour cacher toute leur fragilité et leur mal de vivre :

LOLA LEE - Ça me déprime, icitte !

MARCEL GÉRARD - T'as pas toujours dit ça...

LOLA LEE - J'ai travaillé ici juste parce que j'étais dans la rue...

LA DUCHESSE - Toujours aussi charmante !

LOLA LEE - Toujours aussi grosse !

HOSANNA - A'l'a déjà travaillé ici, c't'agrès-là ?

MARCEL GÉRARD - A'l a été la vedette maison pendant presque un an...

LA DUCHESSE - Vous avez jamais entendu parler de la revue Douze femme, un homme?

Ben, l'homme c'tait elle !

LOLA LEE - Ouan, pis les douze femmes, c'tait toi $!^{14}$

Dans ce parcours sentimental, Rita se souvient des débuts de sa carrière. Il lui a fallu, en effet, douze ans pour s'imposer sur La Main et prendre la vedette du spectacle du Bolivar Lounge sous le pseudonyme de Lola Lee. Avant cette consécration, Lola a dû faire ses classes dans le bordel de Betty Bird où elle a remporté beaucoup de succès sous le surnom de Marigold. Le chemin parcouru, peut-être objet du mépris pour plusieurs, a permis à Lola de remplacer l'aliénation par l'identité d'une star. L'arrivée inopinée de Louise, future Lyla Jasmin, dans la salle de Bolivar Lounge, concrétise les appréhensions de Lola qui perçoit chaque nouvelle venue comme une menace à son règne fragile. L'action commence au moment où Louise Tétrault décide de suivre les traces de son aînée. Ici, la me-

14 Ibid., p. 118-119. 
nace est d'autant plus grande que, subjuguée par sa sœur, Louise en est la copie conforme, en plus jeune :

LOUISE - J'chante la même chose que toé ! Ça va être facile ! Pis j'chante pareil comme toé !

LOLA LEE - Mais es-tu après virer folle ? T'as quand même pas envie de faire carrière en m'imitant !

LOUISE - Ben quoi, y'en a des sœurs qui chantent ! ${ }^{15}$

Devant l'attitude de Louise qui croit naïvement que sa sœur plus âgée lui ouvrira les bras et la détermination de devenir une vedette comme sa sœur que Lola décide d'employer les moyens du mépris. C'est à cause de l'échec argumentatif au Meat Rack, où Louise n'est pas encore convaincue d'abandonner son projet de devenir artiste et de retourner chez sa mère que Lola, désespérée que ses efforts ne la conduisent à rien, décide de lui faire visiter le bordel de Betty Bird. Une fois encore, elle ne parvient pas à atteindre son objectif. En effet, comme le remarque Jean-Marc Barette dans son analyse des personnages tremblayens, « Louise est davantage dégoûtée de sa sœur qui veut se débarrasser d'elle que des gens qu'elle y rencontre ${ }^{16}$. D'ailleurs, elle dit ouvertement à Lola que son èthos a subi une dépréciation par rapport à l'image idéalisée qu'elle s'en avait créée : « Si tu voulais m'écœurer avec toutes tes histoires, t'as réussi ! Mais c'est pas c'que tu m'as montré qui m’écœure, c'est toi ! $\gg{ }^{17}$. Contrairement à ce qui était prévu par Lola et aux techniques dissuasives entreprises, sa sœur découvre progressivement « la camaraderie unissant chanteurs et danseurs (...) qui serrent les coudes afin de passer à travers les épreuves que recèle leur existence $»^{18}$. En outre, le dessein de Lola a un effet contraire et inattendu, parce que Betty joue double jeu et se venge de Lola qui l'avait laissé tomber autrefois. Pour le faire, elle dévoile à Louise les manigences de Lola.

Dans Demain matin, Montréal m'attend, tout comme dans d'autres pièces de Tremblay, Hosanna et Bonjour là, bonjour, le protagoniste, après avoir parcouru une longue recherche de son identité, décide de choisir le chemin qui lui convient le mieux, sans se soucier comment les autres le perçoivent. En choisissant pour ses héros des artistes de show, de cabarets, Tremblay inverse délibérément les notions de succès et de réussite artistique. Les héros de Demain matin ne réalisent jamais leurs rêves, par conséquent, « à défaut de vivre une vie de rêve, ils rêvent leur vie $»^{19}$. Dans cette optique, le plan rusé de Lola Lee est voué à l'échec, sa rhétorique de répulsion par de mauvaises images du milieu artistique montréalais est réduit à néant parce que Louise, au lieu de poursuivre à tout prix la gloire, choisit la recherche de l'identité artistique et personnelle, bien que celle-ci soit méprisée par ceux qui aspirent à la réalisation de l'american dream modèle. En insistant sur une double portée d'Hosanna, Tremblay, lui, explique qu'il s'agit là non seulement de l'identité personnelle de l'individu considéré comme une unité isolée, mais, dans le contexte de la Révolution tranquille en cours, d'une tentative

\footnotetext{
15 Ibid., p. 110.

16 J-M. Barette, L’Univers Michel Tremblay. Dictionnaire des personnages, Montréal 1996, p. 168.

17 M. Tremblay, op. cit., p. 142.

18 G. Desmeules, Ch. Lahaie, op. cit., p. 28.

19 L. Boulanger, op. cit., p. 45.
} 
de déterminer le caractère national du peuple québecois qui « pendant 300 ans, rêvait d'être quelqu'un d'autre au lieu de rêver d'être lui-même $»^{20}$. Cette double dimension de l'histoire présentée est aussi observée dans À toi, pour toujours, ta Marie-Lou, une pièce de Tremblay qui a été créée pendant la crise d'Octobre et qu'on considère « comme une métaphore de la situation politique québécoise $»^{21}$. La recherche d'identité personnelle et nationale est, effectivement, « un thème essentiellement universel » des pièces de Tremblay. Demain matin, Montréal $m$ 'attend porte le même message : «Peu importe ce que les autres pensent de toi et de ta façon de vivre, ce qui compte, c'est qui tu es - quelqu'un d'honnête et de courageux $»^{22}$. C'est justement cette « affirmation de soi » qui « coïncide avec le bouleversement des mentalités par la Révolution tranquille $»^{23}$.

Ainsi, loin de donner l'existence à une dramaturgie nationaliste, Tremblay crée " une dramaturgie nationale authentique $»^{24}$, en permettant à ses personnages d'exprimer « les frustrations accumulées depuis trois cent ans et la révolte qu'elles ont finalement provoquée $»^{25}$. Le parcours de Lola Lee et de sa sœur Louise, au lieu de générer du mépris pour les protagonistes et leur mode de vie, devient le noyau de la recherche de sa propre identité artistique. À travers des métaphores d'ordre quasi almodovarien, la pièce de Tremblay représente sa dramaturgie nationale québécoise, où le protagoniste, allégorie de toute une société, subit une évolution qui lui permet d'apprécier son identité au lieu de la dédaigner, voire mépriser.

Demain matin, Montréal m'attend constitue une métaphore de la vie, qui n'est jamais méprisable, car elle permet d'accepter son identité. On peut ainsi répéter après Nathalie Katinakis que « l'une des forces [de Michel Tremblay], c'est faire rire tout en dénonçant, rendre beau le plus misérable $»^{26}$. Dans cette pièce, le mépris revêt une valeur argumentative. Tout en présentant l'univers artistique qui, dans la société, peut parfois éveiller du mépris, Tremblay entre en jeu avec ses lecteurs et, parait-il, atteint son objectif majeur, il redéfinit et réévalue les notions communes de l'humanité et du parcours que l'individu entreprend pour établir son identité. Le critique Christian Saint-Pierre remarque à ce propos une certaine universalité et intemporalité dans la construction des protagonistes de la pièce. En effet, comme le souligne Saint-Pierre, « tout en étant indissociables de leur époque, ils s'adressent franchement à la nôtre $»^{27}$. Quelle que soit la condition

${ }^{20}$ Canadian Theatre Encyclopedia, http://www.canadiantheatre.com/dict.pl?term=Hosanna (page consultée le 04.04.2018).

21 L. Boulanger, op. cit., p. 51.

22 E.F. Nardocchio, Theatre and Politics in Modern Québec, Edmonton 1986, p. 72.

23 M. Greffard, J.-G. Sabourin, op. cit., p. 79.

24 J. Cotnam, Le Théâtre québecois. Instrument de contestation sociale et politique, Montréal 1976, p. 101.

25 Ibid.

26 N. Katinakis, Critique : Demain Matin, Montréal M'attend au Théâtre du Nouveau Monde, www.musicalavenue.fr (accès : 18.10.2019).

27 Ch. Saint-Pierre, Demain matin, Montréal m'attend : gravir les échelons « Le Devoir » 17 juin 2017, https://www.ledevoir.com/culture/theatre/501473/demain-matin-montreal-m-attend (accès: 1.02.2020). 
où ils vivent, les personnages du drame de Tremblay créent leur destin et réalisent leurs rêves. Même si l'entourage ne leur est pas toujours favorable, le parcours de Lola Lee et de sa sœur cadette prouve que ces protagonistes, loin d'être méprisables s'avèrent « des diamants noirs, des perles dans la fange, des étoiles filantes dans la plus sombre des nuits $»^{28}$.

\section{Bibliographie}

Barette J.-M., L"Univers Michel Tremblay. Dictionnaire des personnages, Montréal 1996.

Boulanger L., Pièces à conviction. Entretiens avec Michel Tremblay, Ottawa 2001.

Canadian Theatre Encyclopedia, http://www.canadiantheatre.com/dict. pl?term=Hosanna (page consultée le 04.04.2018).

Cotnam J., Le Théâtre québecois. Instrument de contestation sociale et politique, Montréal 1976.Desmeules G., Lahaie Ch., Les Personnages du théâtre québecois, Québec 2000.

Desmeules G., Lahaie Ch., Les Personnages du théâtre québecois, Québec 2000.

Greffard M., Sabourin J.-G., Le Théâtre québecois, Québec 1997.

Katinakis N., Critique : Demain Matin, Montréal M'attend au Théâtre du Nouveau Monde, www.musicalavenue.fr (accès : 18.10.2019).

Nardocchio E.F., Theatre and Politics in Modern Québec, Edmonton 1986.

Perelman Ch., L"Empire rhétorique, Paris 2012.

Reboul O., Introduction à la rhétorique, Paris 1995.

Saint-Pierre Ch., Demain matin, Montréal m'attend : gravir les échelons, « Le Devoir » 2017, 17 juin, version numérique, https://www.ledevoir.com/culture/theatre/501473/demain-matin-montreal-m-attend (accès: 1.02.2020).

Tremblay M., Demain matin, Montréal m"attend, Théâtre II, Montréal 2006.

28 Ibid. 
